\title{
Effect of external applied electric field on the silicon solar cell's thermodynamic efficiency
}

\author{
R. Zieba Falama ${ }^{1,2} \cdot$ Justin Mibaile $^{3}$ E. Guemene Dountio ${ }^{2} \cdot$ Noël Djongyang $^{1} \cdot$ \\ Serge Y. Doka ${ }^{4} \cdot$ Timoleon C. Kofane ${ }^{5}$
}

Received: 22 January 2017/Accepted: 10 March 2017/Published online: 22 March 2017

(c) The Author(s) 2017. This article is an open access publication

\begin{abstract}
This paper presents a possible solution to improve the efficiency of photovoltaic solar cells. An external electric field is applied on a silicon photovoltaic solar cell, inducing band-trap ionization of charge carriers. Output current is then monitored and the thermodynamic efficiency is calculated. Results show on the one hand a significant increase in efficiency for a certain margin of applied electric field, and on the another hand the instabilities of efficiency. A simple approach is then suggested for the implementation of these results. An efficiency of $67 \%$ has been reached for an applied electric of $1586 \mathrm{~V} /$ $\mathrm{Cm}$.
\end{abstract}

Keywords Improve efficiency · External applied electric field · Band-trap ionization of charge carriers · Silicon solar cell

\section{Abbreviation \\ $h \quad$ Planck's constant $\left(6.626 \times 10^{-34} \mathrm{Js}\right)$ \\ c Photons propagation speed $\left(3 \times 10^{8} \mathrm{~m} / \mathrm{s}\right)$ \\ $q \quad$ Charge of electron $\left(1.6 \times 10^{-19} \mathrm{C}\right)$}

R. Zieba Falama

rubenziebafalama@gmail.com

1 Department of Renewable Energy, The Higher Institute of the Sahel, University of Maroua, P.O Box 46, Maroua, Cameroon

2 Laboratory of Energy Research, Institute of Geological and Mining Research, P.O Box 4110, Yaoundé, Cameroon

3 Department of Physics, Higher Teachers' Training College, University of Maroua, P.O Box 55, Maroua, Cameroon

4 Department of Physics, Faculty of Science, University of Ngaoundéré, P.O Box 454, Ngaoundéré, Cameroon

5 Department of Physics, Faculty of Science, University of Yaoundé I, P.O. Box. 812, Yaoundé, Cameroon

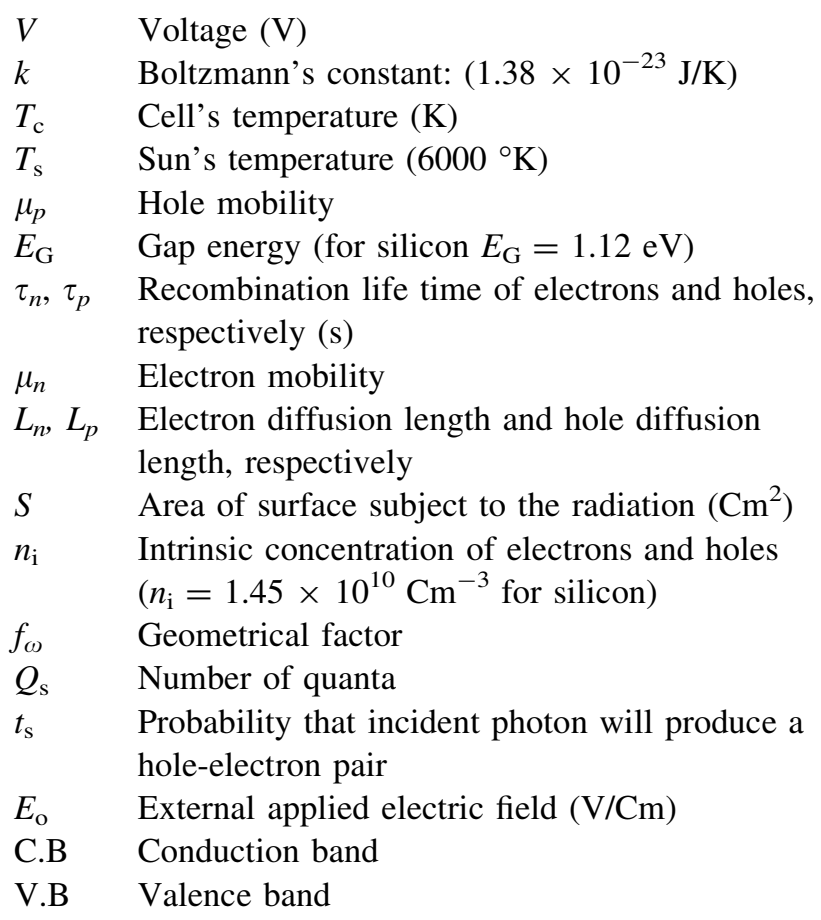

\section{Introduction}

Developing new concepts to improve the efficiency of photovoltaic solar cells is a well-known challenge for the scientific community. In 1961 Shockley and Queisser [1] brought out the theoretical limit of a photovoltaic solar cell. The results of their study are worldwide recognized as theoretical limit of efficiency for a single $p n$-junction solar cell. After them, many studies have been carried out to explore the possibilities of exceeding this limit. Different technologies and methods were used for that purpose [2-15]. Among these approaches, we could cite tandem 
cells, concentrator cell, carrier multiplication, down conversion, hot carriers, etc.

In 1997 , by considering the impact ionization phenomenon to generate hot electrons, Würfel [11] found a maximum efficiency of $85 \%$ for a vanishing band gap of the solar cell. In 1993, Landsberg et al. [12] reported an efficiency of $60.3 \%$ at $E_{\mathrm{G}}=0.8 \mathrm{eV}$ for a solar cell submitted to band-band impact ionization effect. By considering the impact ionization effects on the efficiency of intermediate band solar cells, Gorji [16] has obtained a thermodynamic efficiency of $81.2 \%$, which was higher than the maximum efficiency of $63.2 \%$ for an intermediate band without impact ionization mechanism. All these results show that the impact ionization phenomenon is very promising for the improvement of solar cell efficiency.

Impact ionization is a process in which a charge carrier with high kinetic energy collides with a second charge carrier transferring its kinetic energy to the latter which is hereby lifted to higher energy level [17]. This process increases the number of charge carriers. There are many impact ionization models. One can have: the one carrier model and the two carrier models which are often classified as band-band and band-trap impact ionization [18]. The current study focuses on the band trap impact ionization of the solar cell to reach a high efficiency. Free carriers are subject to trapping [19]. The default and the presence of some impurities in the solar cell material introduce trap levels into the band gap. These levels can emit an electron towards conduction band (case A in Fig. 1), receive an electron from valence band (case $\mathrm{B}$ in Fig. 1), receive an electron from conduction band (case $\mathrm{C}$ in Fig. 1) or loss an electron towards valence band (case D in Fig. 1). The cases $\mathrm{A}$ and $\mathrm{B}$ are those on which this paper focused because they permit to generate additional free charge carriers. These cases can easily been obtained through the process of impact ionization induced by an external source of energy. The current study considers an external applied electric field as the parameter which induces the impact ionization. The models of generation-recombination mechanism with band trap impact ionization involving electrons and holes are presented in Refs. [18, 20-23].

A single $p n$-junction of the solar cell is considered in this work. In Ref. [17] it has been shown that outside an electron diffusion length $L_{n}$ to the right or a hole diffusion

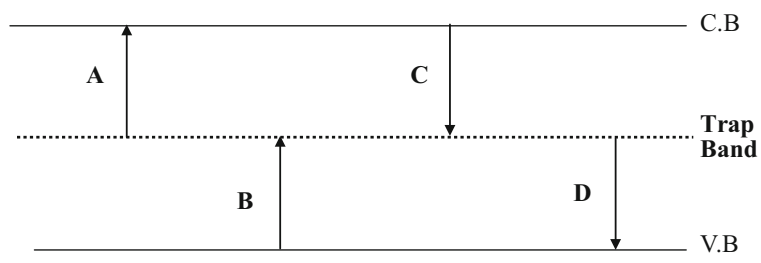

Fig. 1 Transition of charge carriers via trap level length $L_{p}$ to the left of the pn-junction, the charge current through a $p n$-junction is a pure electron current in the $n$ region and a pure hole current in the $p$-region. This charge current is then given by integrating over the contributions to the electron current (alternatively, the contributions to the hole current). Knowing the number of free electrons in $n$-region (or free holes in $p$-region) could be sufficient to evaluate the charge current through a $p n$-junction and thus the open circuit voltage. In this paper, the number of free electrons in $n$-region of the $p n$-junction is evaluated by solving the reaction diffusion equation. This equation is solved with the factorization method. The total hole-electron generation rate due to the solar radiation is evaluated by following the Shockley-Queisser approach [1].

\section{Studied model}

The current delivered by a photovoltaic solar cell is based on the generation-recombination mechanism. The generation-recombination model in this paper is based on the band trap impact ionization phenomenon involving two carriers (holes and electrons). The rate equations of the model are:

$f_{n}(n, p)=Y+\left[X_{1} N_{D}^{*}-X_{1} n-\left(B-X_{1}\right) p\right] n$

$f_{p}(n, p)=Y+\left[X_{2} P_{D}-X_{2} p-\left(B-X_{2}\right) n\right] p$

where $n$ and $p$ are the number of free electrons and free holes, respectively. The variables $X_{1}$ and $X_{2}$ are band-trap impact ionization coefficients (to generate additional electrons and holes, respectively) which depend on the applied electric field. $Y$ and $B$ are the band-band generation coefficient and the band-band recombination coefficient, respectively. $Y$ is a photo-generation parameter due to the illumination of solar cell. This variable is evaluated through Eq. (43). The constants $N_{D}^{*}$ and $N_{\mathrm{t}}$ are the effective donor density and the trap density, respectively. $P_{D}=N_{\mathrm{t}}-N_{D}^{*}$.

Considering the fact that the total charge current through a $p n$-junction is a pure electron current in the $n$-region, the knowledge of the free electrons number in the $n$-region is requested to determine the total charge current through the $p n$-junction. Considering only the transverse direction and neglecting the transverse electric field, the number of free electrons can be determined using the reaction-diffusion equation presented in Eq. (2).

$\frac{\partial n}{\partial t}-D_{n} \frac{\partial^{2} n}{\partial x^{2}}=f_{n}(n, p)$

In steady state regime, $\frac{\partial n}{\partial t}=0$. Thus Eq. (2) turns to:

$\frac{\partial^{2} n}{\partial x^{2}}+\frac{f_{n}(n, p)}{D_{n}}=0$ 
the constant $D_{n}$ is the electron diffusion coefficient defined by:

$D_{n}=\mu_{n} \frac{k T_{\mathrm{c}}}{q}$

\section{Thermodynamic efficiency calculation}

\section{Resolution of the reaction-diffusion equation}

The factorization method $[24,25]$ is used in this work to solve the reaction-diffusion equation. The equation to solve is Eq. (3).

By setting

$$
D=\frac{\partial}{\partial x}, D^{2}=\frac{\partial^{2}}{\partial x^{2}}, g_{1}(n, p)=0, \text { and } f_{1}(n, p)=\frac{f_{n}(n, p)}{D_{n}},
$$

The Eq. (3) can turn to:

$$
\left(D^{2}+g_{1}(n, p) D+\frac{f_{1}(n, p)}{n}\right) n=0
$$

The Eq. (5) can be factorized as:

$$
\left(D-\psi_{12}\right)\left(D-\psi_{11}\right) n=0
$$

where

$f_{1}(n, p)=n \psi_{12} \psi_{11}$

The Eq. (3) can be developed and leads to:

$n^{\prime \prime}-\left(\psi_{11}+\psi_{12}+n \frac{\partial \psi_{11}}{\partial n}\right) n^{\prime}+\psi_{12} \psi_{11} n=0$

The comparison of Eqs. (3) and (8) leads to:

$0=-\left(n \frac{\partial \psi_{11}}{\partial n}+\psi_{11}+\psi_{12}\right)$

$\frac{f_{n}(n, p)}{D_{n}}=\psi_{12} \psi_{11} n$

Eq. (1a) leads to:

$$
\begin{aligned}
\frac{f_{n}(n, p)}{D_{n}}= & \frac{1}{K_{1} D_{n}}\left(1-\frac{\left(B-X_{1}\right) p}{\frac{Y}{n}+X_{1}\left(N_{D}^{*}-n\right)}\right) \\
& \times K_{1}\left(\frac{Y}{n}+X_{1}\left(N_{D}^{*}-n\right)\right) n
\end{aligned}
$$

Therefore, referring to Eq. (10), $\psi_{i j}$ could be choosing such as:

$$
\begin{aligned}
& \psi_{11}=K_{1}\left(\frac{Y}{n}+X_{1}\left(N_{D}^{*}-n\right)\right) \\
& \psi_{12}=\frac{1}{K_{1} D_{n}}\left(1-\frac{\left(B-X_{1}\right) p}{\frac{Y}{n}+X_{1}\left(N_{D}^{*}-n\right)}\right)
\end{aligned}
$$

where $K_{1}$ is an arbitrary constant to be determined.To determine the constants $K_{1}$ let us consider Eq. (9). This equation can be developed and leads to,

$K_{1}^{2} X_{1} N_{D}^{*}+\frac{1}{D_{n}}-2 K_{1}^{2} X_{1} n-\frac{\left(B-X_{1}\right) p}{D_{n}\left(\frac{Y}{n}+X_{1} N_{D}^{*}-X_{1} n\right)}=0$

The Eq. (13) admits solution if:

$\left\{\begin{array}{l}K_{1}^{2} X_{1} N_{D}^{*}+\frac{1}{D_{n}}=0, \\ -2 K_{1}^{2} X_{1} n-\frac{\text { or }}{D_{n}\left(\frac{Y}{n}+X_{1} N_{D}^{*}-X_{1} n\right)}=0\end{array}\right.$

The first condition of Eq. (14) leads to:

$K_{1}=i \sqrt{\frac{1}{X_{1} N_{D}^{*} D_{n}}}$,

The Eq. (5) transformed to two possible differential equations of first order such as [25]:

$n^{\prime}-\psi_{11}(n, p) n=0$

$n^{\prime}-\psi_{12}(n, p) n=0$

Let us consider Eq. (16a). The replacement of Eq. (12a) into Eq. (16a) leads to:

$\int \frac{\mathrm{d} n}{Y+X_{1}\left(N_{D}^{*}-n\right) n}=\int_{z_{0}}^{z} K_{1} \mathrm{~d} z$

The Eq. (17) permits to obtain:

$\left|\frac{n-\alpha}{n-\beta}\right|=e^{K_{1} \sqrt{\Delta}\left(z-z_{0}\right)}$

where

$\Delta=\left(X_{1} N_{D}^{*}\right)^{2}+4 Y X_{1}$,

$\alpha=\frac{X_{1} N_{D}^{*}-\sqrt{\Delta}}{2 X_{1}}$,

and

$\beta=\frac{X_{1} N_{D}^{*}+\sqrt{\Delta}}{2 X_{1}}$

We have to consider two cases:

First case

For $\left|\frac{n-\alpha}{n-\beta}\right|=-\frac{n-\alpha}{n-\beta}$, Eq. (18) leads to:

$n=\frac{\alpha}{1+e^{K_{1} \sqrt{\Delta}\left(x-x_{0}\right)}}+\frac{\beta e^{K_{1} \sqrt{\Delta}\left(x-x_{0}\right)}}{1+e^{K_{1} \sqrt{\Delta}\left(x-x_{0}\right)}}$ 
By setting $K_{1}=i K_{2}$, Eq. (20) can turn to:

$$
\begin{aligned}
n= & \frac{\alpha e^{-i K_{2} \sqrt{\Delta}\left(x-x_{0}\right) / 2}}{e^{i K_{2} \sqrt{\Delta}\left(x-x_{0}\right) / 2}+e^{-i K_{2} \sqrt{\Delta}\left(x-x_{0}\right) / 2}} \\
& +\frac{\beta e^{i K_{2} \sqrt{\Delta}\left(x-x_{0}\right) / 2}}{e^{i K_{2} \sqrt{\Delta}\left(x-x_{0}\right) / 2}+e^{-i K_{2} \Delta\left(x-x_{0}\right) / 2}}
\end{aligned}
$$

where

$K_{2}=\sqrt{\frac{1}{X_{1} N_{D}^{*} D_{n}}}$

By setting $\gamma=\frac{\alpha e^{-i K_{2} \sqrt{\Delta}\left(x-x_{0}\right) / 2}}{e^{i K_{2} \sqrt{\Delta}\left(x-x_{0}\right) / 2}+e^{-i K_{2} \sqrt{\Delta}\left(x-x_{0}\right) / 2}}$ and $\delta=$

Second case

For $\left|\frac{n-\alpha}{n-\beta}\right|=\frac{n-\alpha}{n-\beta}$, Eq. (18) leads to:

$n=\frac{\alpha}{1-e^{K_{1} \sqrt{\Delta}\left(x-x_{0}\right)}}-\frac{\beta e^{K_{1} \sqrt{\Delta}\left(x-x_{0}\right)}}{1-e^{K_{1} \sqrt{\Delta}\left(x-x_{0}\right)}}$

Following a same approach like in the first case, one gets:

$n=\frac{N_{D}^{*}}{2}+\frac{\sqrt{\Delta}}{2 X_{1}} \cot \left(\frac{K_{2} \sqrt{\Delta}\left(x-x_{0}\right)}{2}\right)$

$\frac{\beta e^{i K_{2} \sqrt{\Delta}\left(x-x_{0}\right) / 2}}{e^{i K_{2} \sqrt{\Delta}\left(x-x_{0}\right) / 2}+e^{-i K_{2} \sqrt{\Delta}\left(x-x_{0}\right) / 2}}$, one could have:

$\gamma=\frac{\alpha\left(\frac{e^{i K_{2} \sqrt{\Delta}\left(x-x_{0}\right) / 2}+e^{-i K_{2} \sqrt{\Delta}\left(x-x_{0}\right) / 2}}{2}\right)+\alpha e^{-i K_{2} \sqrt{\Delta}\left(z-z_{0}\right) / 2}-\alpha\left(\frac{e^{i K_{2} \sqrt{\Delta}\left(x-x_{0}\right) / 2}+e^{-i K_{2} \sqrt{\Delta}\left(x-x_{0}\right) / 2}}{2}\right)}{e^{i K_{2} \sqrt{\Delta}\left(x-x_{0}\right) / 2}+e^{-i K_{2} \sqrt{\Delta}\left(x-x_{0}\right) / 2}}$

and

$\delta=\frac{\beta\left(\frac{e^{i K_{2} \sqrt{\Delta}\left(x-x_{0}\right) / 2}+e^{-i K_{2} \sqrt{\Delta}\left(x-x_{0}\right) / 2}}{2}\right)+\beta e^{i K_{2} \sqrt{\Delta}\left(x-x_{0}\right) / 2}-\beta\left(\frac{e^{i K_{2} \sqrt{\Delta}\left(x-x_{0}\right) / 2}+e^{-i K_{2} \sqrt{\Delta}\left(x-x_{0}\right) / 2}}{2}\right)}{e^{i K_{2} \sqrt{\Delta}\left(x-x_{0}\right) / 2}+e^{-i K_{2} \sqrt{\Delta}\left(x-x_{0}\right) / 2}}$

Equations (23a) and (23b) turn to:

$\gamma=\frac{\alpha}{2}\left(1-\tan \left(\frac{K_{2} \sqrt{\Delta}\left(x-x_{0}\right)}{2}\right)\right)$

and

$\delta=\frac{\beta}{2}\left(1+\tan \left(\frac{K_{2} \sqrt{\Delta}\left(x-x_{0}\right)}{2}\right)\right)$

Thus, Eq. (20) turns to:

$$
\begin{aligned}
n= & \frac{\alpha}{2}\left(1-\tan \left(\frac{K_{2} \sqrt{\Delta}\left(x-x_{0}\right)}{2}\right)\right) \\
& +\frac{\beta}{2}\left(1+\tan \left(\frac{K_{2} \sqrt{\Delta}\left(x-x_{0}\right)}{2}\right)\right)
\end{aligned}
$$

By replacing $\alpha$ and $\beta$ by their expressions in Eq. (25) one obtains:

$n=\frac{N_{D}^{*}}{2}-\frac{\sqrt{\Delta}}{2 \mathrm{X}_{1}} \tan \left(\frac{K_{2} \sqrt{\Delta}\left(x-x_{0}\right)}{2}\right)$

\section{Charge current density through the $p n$-junction}

According to Ref. [17], the total charge current through a $p n$-junction could be expressed by:

$j_{\mathrm{Q}}=q \int_{-L_{p}}^{L_{n}} \operatorname{div} j_{n} \mathrm{~d} x$

where

$L_{n}=\sqrt{D_{n} \tau_{n}}$

$L_{p}=\sqrt{D_{p} \tau_{p}}$

$D_{p}=\mu_{p} \frac{k T_{\mathrm{c}}}{q}$

From the continuity equation for electrons, one gets:

$\operatorname{divj}_{n}=q\left(\frac{\partial n}{\partial t}-f_{n}(n, p)\right)$

By replacing Eq. (1a) into Eq. (31) and Eq. (31) into Eq. (29), and considering the fact that $\frac{\partial n}{\partial t}=0$ (steady state condition), then Eq. (29) turns to: 


$$
\begin{aligned}
j_{\mathrm{Q}}= & -q^{2} \int_{-L_{p}}^{L_{n}} Y \mathrm{~d} x-q^{2} X_{1} N_{D}^{*} \int_{-L_{p}}^{L_{n}} n \mathrm{~d} x+q^{2} X_{1} \int_{-L_{p}}^{L_{n}} n^{2} \mathrm{~d} x \\
& +q^{2}\left(B-X_{1}\right) \int_{-L_{p}}^{L_{n}} p n \mathrm{~d} x
\end{aligned}
$$

For simplicity let us set:

$$
\begin{aligned}
& \theta_{1}=q^{2} \int_{-L_{p}}^{L_{n}} Y \mathrm{~d} x, \\
& \theta_{2}=q^{2} X_{1} N_{D}^{*} \int_{-L_{p}}^{L_{n}} n \mathrm{~d} x, \\
& \theta_{3}=q^{2} X_{1} \int_{-L_{p}}^{L_{n}} n^{2} \mathrm{~d} x, \\
& \theta_{4}=q^{2}\left(B-X_{1}\right) \int_{-L_{p}}^{L_{n}} p n \mathrm{~d} x
\end{aligned}
$$

The calculation of $\theta_{1}, \theta_{2}, \theta_{3}$ and $\theta_{4}$ leads to:

$$
\theta_{1}=q^{2} Y\left(L_{n}+L_{p}\right)
$$

The short-circuit current $j_{\mathrm{sc}}$ (when $V=0$ ) is defined as:

$j_{\mathrm{sc}}=-\theta_{1}-\theta_{2}+\theta_{3}+q^{2} n_{\mathrm{i}}^{2}\left(B-X_{1}\right)\left(L_{n}+L_{p}\right)$

Equation (35) could be rewritten as:

$j_{\mathrm{Q}}=j_{\mathrm{sc}}+q^{2} n_{\mathrm{i}}^{2}\left(B-X_{1}\right)\left(L_{n}+L_{p}\right)\left(\exp \left(\frac{q V}{k T_{\mathrm{c}}}\right)-1\right)$

By considering the dark where $j_{\mathrm{sc}}=0$ and for large negative voltages (where $\exp \left(\frac{q V}{k T_{\mathrm{c}}}\right) \ll 1$ ), one gets the reverse saturation current $j_{\mathrm{s}}$ as:

$j_{\mathrm{s}}=q^{2} n_{\mathrm{i}}^{2}\left(B-X_{1}\right)\left(L_{n}+L_{p}\right)$

From the relation

$j_{\mathrm{Q}}=j_{\mathrm{sc}}+j_{\mathrm{s}}\left(\exp \left(\frac{q V}{k T_{\mathrm{c}}}\right)-1\right)$,

the open circuit voltage (when $j_{\mathrm{Q}}=0$ ) is deducted as:

$V_{\mathrm{oc}}=\frac{k T_{\mathrm{c}}}{q} \ln \left(1-\frac{j_{\mathrm{sc}}}{j_{\mathrm{s}}}\right)$

According to Ref. [17], the thermodynamic efficiency is given by:

$\eta_{\text {thermodynamic }}=\frac{q V_{\mathrm{oc}}}{E_{\mathrm{G}}+3 k T_{\mathrm{c}}}$

According to Ref. [1], the photo-generation rate of holeelectron pairs $Y$ is defined by:

$$
\begin{array}{r}
\theta_{2}=q^{2} X_{1} N_{D}^{*}\left(\frac{N_{D}^{*}}{2}\left(L_{n}+L_{p}\right)+\frac{1}{K_{2} X_{1}} \log \left|\cos \left(\frac{K_{2} \sqrt{\Delta}}{2}\left(L_{n}-x_{0}\right)\right)\right|-\frac{1}{K_{2} X_{1}} \log \left|\cos \left(\frac{K_{2} \sqrt{\Delta}}{2}\left(-L_{p}-x_{0}\right)\right)\right|\right) \\
\theta_{3}=q^{2} X_{1}\left(\begin{array}{r}
\left.\left(\frac{N_{D}^{* 2}}{4}-\frac{\Delta}{4 X_{1}^{2}}\right)\left(L_{n}+L_{p}\right)+\frac{N_{D}^{*}}{K_{2} X_{1}} \log \left|\cos \left(\frac{K_{2} \sqrt{\Delta}}{2}\left(L_{n}-x_{0}\right)\right)\right|-\frac{N_{D}^{*}}{K_{2} X_{1}} \log \left|\cos \left(\frac{K_{2} \sqrt{\Delta}}{2}\left(-L_{p}-x_{0}\right)\right)\right|\right) \\
+\frac{\sqrt{\Delta}}{2 K_{2} X_{1}^{2}} \tan \left(\frac{K_{2} \sqrt{\Delta}}{2}\left(L_{n}-x_{0}\right)\right)-\frac{\sqrt{\Delta}}{2 K_{2} X_{1}^{2}} \tan \left(\frac{K_{2} \sqrt{\Delta}}{2}\left(-L_{p}-x_{0}\right)\right)
\end{array}\right)
\end{array}
$$

$$
\theta_{4}=q^{2} n_{\mathrm{i}}^{2}\left(B-X_{1}\right)\left(L_{n}+L_{p}\right) \exp \left(\frac{q V}{k T_{\mathrm{c}}}\right)
$$

Thus, Eq. (32) could be rewritten as:

$$
j_{\mathrm{Q}}=-\theta_{1}-\theta_{2}+\theta_{3}+q^{2} n_{\mathrm{i}}^{2}\left(B-X_{1}\right)\left(L_{n}+L_{p}\right) \exp \left(\frac{q V}{k T_{\mathrm{c}}}\right)
$$

$Y=S f_{\omega} t_{\mathrm{s}} Q_{\mathrm{s}}$

where

$$
\begin{aligned}
& Q_{\mathrm{s}}=\left[2 \pi\left(k T_{\mathrm{s}}\right)^{3} /\left(h^{3} c^{2}\right)\right] \int_{x_{\mathrm{g}}}^{\infty} \frac{x^{2} \mathrm{~d} x}{e^{x}-1} \\
& x_{\mathrm{g}}=\frac{E_{\mathrm{G}}}{k T_{\mathrm{s}}}
\end{aligned}
$$


Table 1 Typical materials parameters corresponding to $\alpha$-si near room temperature for the $\mathrm{g}-\mathrm{r}$ process of band-trap impact ionization [18]

\begin{tabular}{ll}
\hline Parameters & Value \\
\hline$X_{l}$ & $3 \times 10^{-5} \exp \left(-2 \times 10^{4} / E_{\mathrm{o}}\right) \mathrm{Cm}^{3} \mathrm{~S}^{-1}$ \\
$B$ & $10^{-10} \mathrm{Cm}^{3} \mathrm{~S}^{-1}$ \\
$N_{D}^{*}$ & $2 \times 10^{15} \mathrm{Cm}^{-3}$ \\
$N_{\mathrm{t}}$ & $3 \times 10^{15} \mathrm{Cm}^{-3}$ \\
$\mu_{n} / \mu_{p}$ & 1 \\
$\mu_{n}$ & $2 \mathrm{Cm}^{2} / \mathrm{Vs}$ \\
\hline
\end{tabular}

\section{Discussion}

All the needed parameters for simulation are presented in Table 1.

Figure 2 presents the thermodynamic efficiency of the studied silicon solar cell modeled as an increase function for the external applied electric field in the range of $0-1586 \mathrm{~V} / \mathrm{Cm}$. An efficiency of $67 \%$ is reached for $E_{\mathrm{o}}=1586 \mathrm{~V} / \mathrm{Cm}$. From $E_{\mathrm{o}}>1586 \mathrm{~V} / \mathrm{Cm}$ an efficiency fluctuation (increasing and decreasing) is noted. This fluctuation could be due to current instabilities which emerge from solar cells (which are semiconductors) when the applied electric field is increasing [26]. Figure 3 has been plotted for $0<E_{\mathrm{o}}<10^{6} \mathrm{~V} / \mathrm{Cm}$ and shows that the solar cell could reach a high efficiency for strong electric field as it is the case for $E_{\mathrm{o}}=8 \times 10^{5} \mathrm{~V} / \mathrm{Cm}$ where an efficiency of $72.7 \%$ has been reached. In this case, the problem is that, for the same value of electric field, there are different values of efficiency because of fluctuations. Therefore, it could be difficult to determinate exactly the high efficiency of the solar cell subjected to impact ionization (induced by an external applied electric field) in the margin of which efficiency fluctuates and is unstable. The results obtained shows that the band-trap impact ionization of charge carriers induced by an applied electric field could be an interesting solution to reach a high efficiency of the photovoltaic solar cells. However, it is very important to know and avoid applying electric field belonging to the range which induces efficiency instabilities. Theoretically a high efficiency of solar cell could be reached even for applied electric fields of average intensity $(67 \%$ has been reached at $\left.E_{\mathrm{o}}=1586 \mathrm{~V} / \mathrm{Cm}\right)$.

\section{Conclusion}

In this paper, the effect of an external applied electric field on the thermodynamic efficiency of a silicon photovoltaic solar cell has been studied. Theoretically, it has been shown that an auxiliary applied electric field could be a very promising solution to reach a high efficiency
Fig. 2 Thermodynamic efficiency versus external applied electric field for $0<E_{\mathrm{o}}<10^{4} \mathrm{~V} / \mathrm{Cm}$.

$T_{\mathrm{c}}=300 \mathrm{~K}, T_{\mathrm{s}}=6000 \mathrm{~K}$,

$S=1 \mathrm{Cm}^{2}, f_{\omega}=2.18 \times 10^{-5}$,

$t_{\mathrm{s}}=1, \tau_{n}=\tau_{p}=10^{-6} \mathrm{~s}$,

$x_{0}=0$

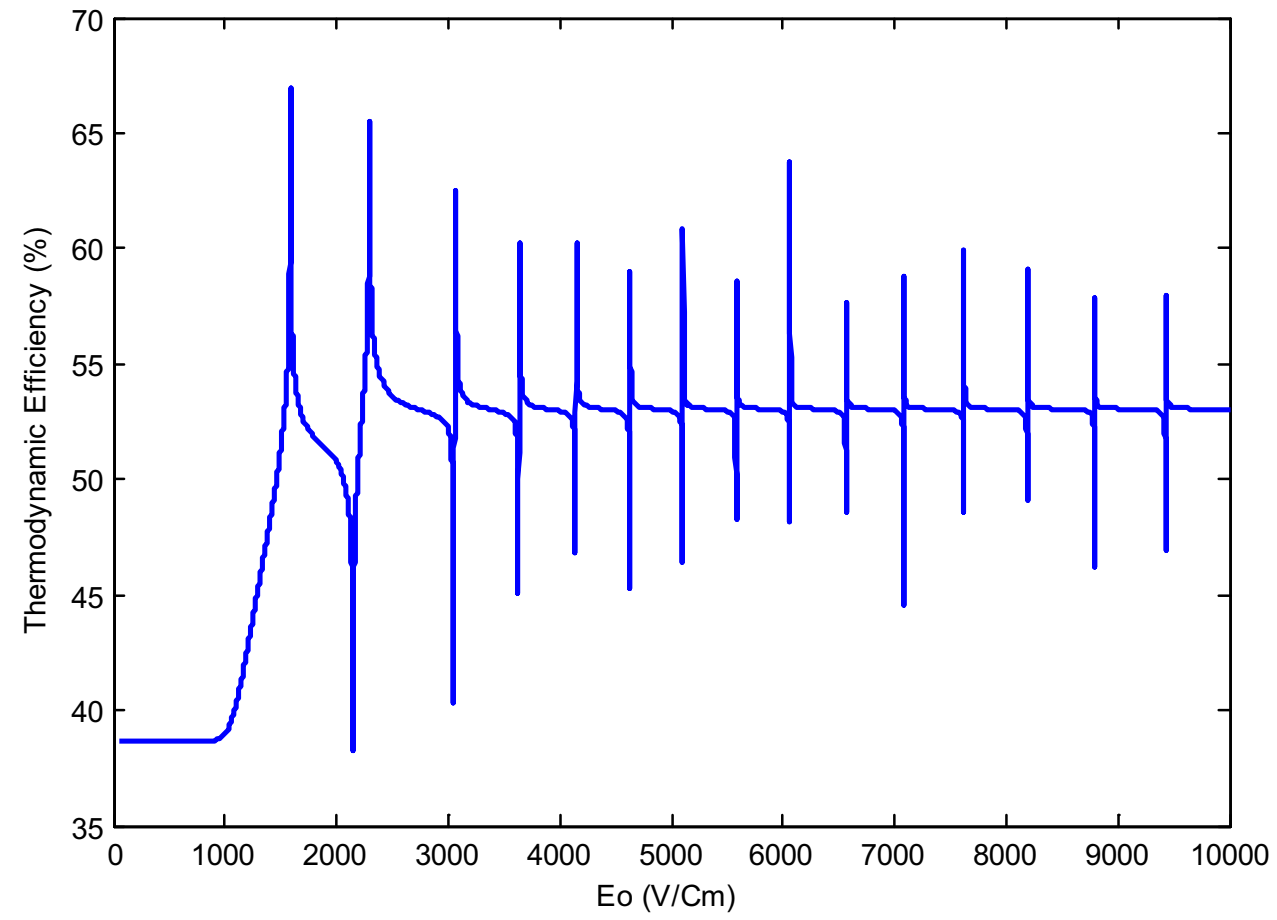


Fig. 3 Thermodynamic efficiency versus external applied electric field for $0<E \mathrm{o}<10^{6} \mathrm{~V} / \mathrm{Cm}$.

$T_{\mathrm{c}}=300 \mathrm{~K}, T_{\mathrm{s}}=6000 \mathrm{~K}$,

$S=1 \mathrm{Cm}^{2}, f_{\omega}=2.18 \times 10^{-5}$,

$t_{\mathrm{s}}=1, \tau_{n}=\tau_{p}=10^{-6} \mathrm{~s}$,

$x_{0}=0$

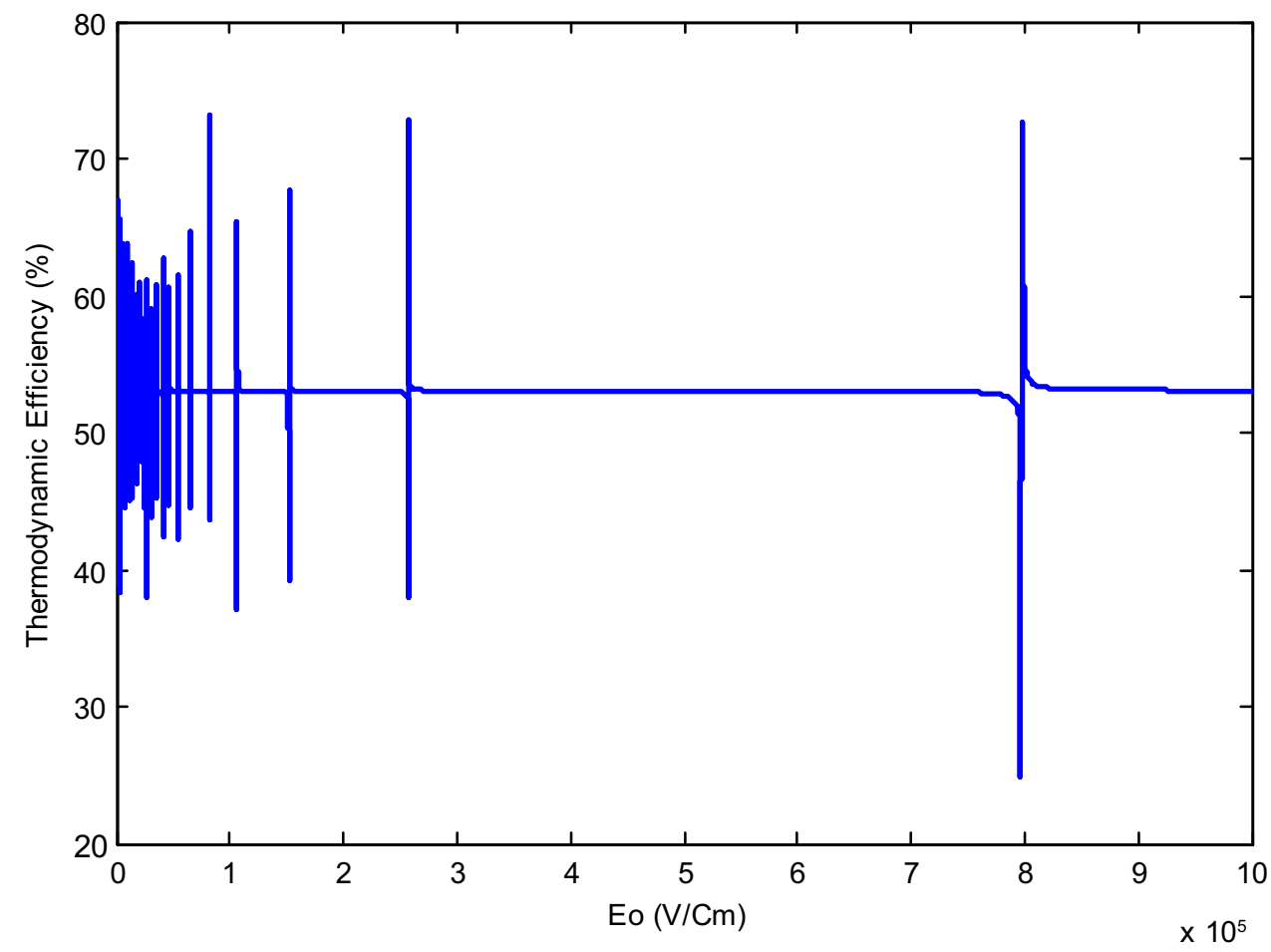

of the solar cells. However, it is not always the stronger electric field which is necessary to induce the higher efficiency. There are efficiency instabilities for strong applied electric field to solar cells.

Acknowledgements The authors thank Taku Agbor Junior for his useful comments.

Open Access This article is distributed under the terms of the Creative Commons Attribution 4.0 International License (http://crea tivecommons.org/licenses/by/4.0/), which permits unrestricted use, distribution, and reproduction in any medium, provided you give appropriate credit to the original author(s) and the source, provide a link to the Creative Commons license, and indicate if changes were made.

\section{References}

1. Shockley, W., Queisser, H.J.: Detailed balance limit of efficiency of p-n junction solar cells. J. Appl. Phys. 32(3), 510-519 (1961)

2. Alharbi, F.H., Kais, S.: Theoretical limits of photovoltaics efficiency and possible improvements by intuitive approaches learned from photosynthesis and quantum coherence. Renew. Sustain. Energy Rev. 43, 1073-1089 (2015)

3. Ze'ev, R.A., Gharghi, M., Niv, A., Gladden, C., Zhang, X.: Theoretical efficiency of 3rd generation solar cells: comparison between carrier multiplication and down-conversion. Sol. Energy Mater. Sol. Cells 99, 308-315 (2012)

4. Guter, W., et al.: Current-matched triple junction solar cell reaching $41.1 \%$ conversion efficiency under concentrated sunlight. Appl. Phys. Lett. 94(22), 223504 (2009)

5. Markvart, T.: Thermodynamics of losses in photovoltaic conversion. Appl. Phys. Lett. 91(6), 064102 (2007)
6. Imenes, A.G., Mills, D.R.: Spectral beam splitting technology for increased conversion efficiency in solar concentrating systems: a review. Sol. Energy Mater. Sol. Cells 84(1), 19-69 (2004)

7. Brown, A.S., Green, M.A.: Intermediate band solar cell with many bands: ideal performance. J. Appl. Phys. 94(9), 6150-6158 (2003)

8. Brown, A.S., Green, M.A., Corkish, R.P.: Limiting efficiency for a multi-band solar cell containing three and four bands. Phys. E: Low. Dimens. Syst Nanostruct. 14(1), 121-125 (2002)

9. Anderson, N.G.: On quantum well solar cell efficiencies. Phys. E: Low. Dimens. Syst. Nanostruct. 14(1), 126-131 (2002)

10. De Vos, A., Desoete, B.: On the ideal performance of solar cells with larger-than-unity quantum efficiency. Sol. Energy. Mater. Sol. Cells. 51(3), 413-424 (1998)

11. Würfel, P.: Solar energy conversion with hot electrons from impact ionisation. Sol. Energy Mater. Sol. Cells. 46(1), 43-52 (1997)

12. Landsberg, P.T., et al.: Band-band impact ionization and solar cell efficiency. J. Appl. Phys. 74(2), 1451 (1993)

13. Ross, R.T., Nozik, A.J.: Efficiency of hot-carrier solar energy converters. J. Appl. Phys. 53(5), 3813-3818 (1982)

14. De Vos, A.: Detailed balance limit of the efficiency of tandem solar cells. J. Phys. D. Appl. Phys. 13(5), 839 (1980)

15. Werner, J. H., Brendel, R., Oueisser, H. J.: New Upper Efficiency Limits For Semiconductor Solar Cells. IEEE Photovoltaic Specialists Conference. IEEE First World Conference on Photovoltaic Energy Conversion, Conference Record of The Twenty Fourth, vol. 2, pp. 1742-1745. IEEE, New York (1994)

16. Gorji, N.E.: Impact ionization effects on the efficiency of the intermediate band solar cells. Phys. E: Low. Dimens. Syst. Nanostruct. 44(7), 1608-1611 (2012)

17. Würfel, P.: Physics of Solar Cell: From Principles to New Concepts. Wiley VCH Publishers, Weinheim (2005)

18. Schöll, E.: Nonequilibrium phase transition in semiconductor self-organisation induced by generation and recombination processes. Springer, Berlin (1987) 
19. Fonash, J.S.: Solar Cell Device Physics, 2nd edn. Elsevier inc, Amsterdam (2010)

20. Scholl, E., Landsberg, P. T.: Proceeding of the 14th international. conference on the physics of semiconductors (Edinburgh 1978). In: Wilson, B. L. H. (ed.) Institute of Physics Conference Series, vol. 43, pp. 461. Institute of Physics, Bristol (1979)

21. Scholl, E., Landsberg, P. T.:Semiconductor models for first and second order non-equilibrium phase transitions. In: Proceedings of the Royal Society of London A: Mathematical, Physical and Engineering Sciences, vol. 365, pp. 495. The Royal Society, London (1979)

22. Landsberg, P.T., Robbins, D.J., Schöll, E.: Threshold switching as a generation-recombination induced non-equilibrium phase transition. Phys. Status. Solidi. (a) SO. 50(2), 423-426 (1978)
23. Robbins, D.J., Landsberg, P.T., Schöll, E.: Phys. Status. Solidi. (a) 65, 353-364 (1981)

24. Rosu, H.C., Cornejo-Pérez, O.: Super symmetric pairing of kinks for polynomial nonlinearities. Phys. Rev. E. 71, 046600-046607 (2005)

25. Fahmy, E.S.: Exact solutions for some reaction-diffusion systems with nonlinear reaction polynomials terms. Appl. Math. Sci. 3, 533-540 (2009)

26. Schöll, E.: Modeling nonlinear and chaotic dynamics in semiconductor device structure. VLSI, Design. 6, 321-329 (1998) 